\title{
Basal Ganglia Role in Motor Control and Movement Disorders
}

\author{
Behrouz Saberi Tehrani* and Behzad Saberi \\ PHD in preventive and community medicine, Iran
}

*Corresponding author: Behrouz Saberi Tehrani, PHD in preventive and community medicine, Iran.

\author{
Received Date: April 30, 2019 \\ Published Date: May 06, 2019
}

\section{Opinion}

Basal ganglia dysfunction or lesions can result in movement disorders. This anatomical structure which is located in diencephalon and midbrain, consist of some nuclei which are interconnected together in a complex manner. Voluntary and involuntary motor functions would be controlled by basal ganglia which its increased output results in tremulous, rigid and slow movements in comparison with decreased outputstates which cause hyperkinetic movements. Although basal ganglia is responsible for motor function control, but it can also control some other brain functions like motivation, emotions and thoughts. So dysfunction in basal ganglia can also cause some pathologies like attention deficits, obsessions, mood disorders etc. Studying the anatomical and physiological aspects of basal ganglia function, would result in finding new treatment options for various movement disorders including pharmacological and brain stimulation treatment methods.

There are some inputs to the basal ganglia like noradrenergic ones from locus ceruleus, serotonergic ones from raphe nuclei, nigrostriatal, thalamostriatal and striatum-corticostriatal pathways. Striatum outputs include inhibitory GABAergic projections from putamen to substantia nigra pars reticulate, the globus pallidus interna and thalamus and another inhibitory projection from putamen to globus pallidus externa and subthalamic nucleus which by increasing in glutaminergic input to the globus pallidus interna, would inhibit thalamic connections. The dopaminergic nigrostriatal pathway modulates these pathways which are named direct and indirect ones. They have putaminal neurons containing D1 and D2 dopamine receptors. Indirect pathway produces inhibitory effects in comparison with direct pathway which produces excitatory effects on thalamus and its output. GABAergic inhibitory inputs of the thalamus will return to the cerebral cortex in the premotor area. If there would be any dysregulation in the striatal outputs, some pathologies would appear like Parkinson's disease in which inhibitory thalamic output would be increased as a result of striatal dopamine deficiency and globus pallidus interna overactivity in indirect and direct pathways, which lead to rigidity and slowness as the signs of decreasing in motor activity. Globus pallidus interna stimulation would decrease such motor problems in Parkinson's disease. In Huntington's disease, decreasing in inhibitory thalamic output and chorea would be appeared due to indirect and direct pathways dysregulation as a result of decreasing in cholinergic and GABAergic striatal cells. Appearance of Hemiballismus in the pathologies which would affect subthalamic nucleus, is due to decreasing in inhibitory thalamic output. This model of basal ganglia circuitry would explain various hyperkinetic movement disorders and related pathologies, however there should be more studies which can explain some other disorders like tics and dystonia which their mechanisms are not very much clear yet.

\section{Acknowledgement}

None.

\section{Conflict of Interest}

No conflict of interest.

\section{References}

1. Postuma RB, Dagher A (2006) Basal ganglia functional connectivity based on a meta-analysis of 126 positron emission tomography and functional magnetic resonance imaging publications. Cerebral Cortex 16(10): 1508-1521.

2. Chakravarthy VS, Joseph D, Bapi RS (2010) What do the basal ganglia do? A modeling perspective. Biological Cybernetics 103(3): 237-253.

3. Voorn P, Vanderschuren LJ, Groenewegen HJ, Robbins TW, Pennartz CM (2004) Putting a spin on the dorsal-ventral divide of the striatum. Trends in Neurosciences 27(8): 468-474. 
4. Lanciego José L, Luquin Natasha, Obeso José A (2017) Functional Neuroanatomy of the Basal Ganglia. Cold Spring Harbor Perspectives in Medicine 2(12): a009621.

5. Silkis I (2001) The cortico-basal ganglia-thalamocortical circuit with synaptic plasticity. II. Mechanism of synergistic modulation of thalamic activity via the direct and indirect pathways through the basal ganglia. Biosystems 59(1): 7-14.

6. DeLong Mahlon, Wichmann Thomas (2017) Changing Views of Basal Ganglia Circuits and Circuit Disorders. Clinical EEG and Neuroscience 41(2): 61-67.

7. Hélie S, Ell SW, Ashby FG (2015) Learning robust cortico-cortical associations with the basal ganglia: an integrative review. Cortex 64: 123-135.

8. Eisinger RS, Urdaneta ME, Foote KD, Okun MS, Gunduz A (2018) Nonmotor Characterization of the Basal Ganglia: Evidence from Human and
Non-human Primate Electrophysiology. Frontiers in Neuroscience 12: 385 .

9. Cameron IG, Watanabe M, Pari G, Munoz DP (2010) Executive impairment in Parkinson's disease: response automaticity and task switching. Neuropsychologia 48(7): 1948-1957.

10. Calabresi P, Picconi B, Tozzi A, Ghiglieri V, Di Filippo M (2014) Direct and indirect pathways of basal ganglia: a critical reappraisal. Nature Neuroscience 17(8): 1022-1030.

11. Tiago V Maia, Michael J Frank (2017) From Reinforcement Learning Models of the Basal Ganglia to the Pathophysiology of Psychiatric and Neurological Disorders. Nature Neuroscience 14(2): 154-162.

12. DeLong M, Wichmann T (2017) Update on models of basal ganglia function and dysfunction. Parkinsonism \& Related Disorders 15(Suppl 3): S237-S240. 